\title{
Gone Till November: A disagreement in Einstein scholarship*
}

\author{
$\operatorname{Tim} \operatorname{Räz}{ }^{\dagger}$
}

March 5, 2015

\begin{abstract}
The present paper examines an episode from the historiography of the genesis of general relativity. Einstein rejected a certain theory in the so-called "Zurich notebook" in 1912-13, but he reinstated the same theory for a short period of time in the November of 1915 . Why did Einstein reject the theory at first, and why did he change his mind later? The group of Einstein scholars who reconstructed Einstein's reasoning in the Zurich notebook disagree on how to answer these questions. According to the "majority view", Einstein was unaware of so-called "coordinate conditions", and he relied on so-called "coordinate restrictions". John Norton, on the other hand, claims that Einstein must have had coordinate conditions all along, but committed a different mistake, which he would repeat in the context of the famous "hole argument". After an account of the two views, and of the reactions by the respective opponents, I will probe the two views for weaknesses, and try to determine how we might settle the disagreement. Finally, I will discuss emerging methodological issues.
\end{abstract}

\section{Contents}

1 Introduction 2

2 A Bird's Eye View of the Episode 3

3 The Two Views $\quad 7$

3.1 The Majority View . . . . . . . . . . . . . . . . . . . . 7

3.2 Norton on the Majority View . . . . . . . . . . . . . . . 9

3.3 Norton's View . . . . . . . . . . . . . . . . . . . . . . 11

3.4 The Majority on Norton's View . . . . . . . . . . . . . . . . . 14

${ }^{*}$ To appear in: Sauer, T. and Scholl, R., eds. (2015): The Philosophy of Historical Case Studies. Boston Studies in the Philosophy and History of Science. Springer.

${ }^{\dagger}$ Universität Konstanz, FB Philosophie, 78457 Konstanz, Germany. E-mail: tim.raez@gmail.com 
4 How to Resolve the Disagreement

4.1 Einstein's Knowledge of Coordinate Conditions . . . . . . . . 16

4.2 Evidence Against the Majority View: "Vermutlicher Gravitationstensor" . . . . . . . . . . . . . . . . 17

4.3 Theoretical Virtues: Simplicity and Explanatory Power . . . . 18

5 Methodological Issues $\quad 19$

5.1 Reconstructing Einstein . . . . . . . . . . . . . . . 20

5.2 Historical Errors: A Dilemma . . . . . . . . . . . . . . 21

6 Conclusion

\section{Introduction}

The present paper examines an episode from the historiography of general relativity (GR) that exhibits methodological problems of history and philosophy of science. These problems emerge because there are two competing accounts of an important episode from Einstein's long path to GR. ${ }^{1}$ The fact that two competing accounts exist is not particularly exciting in itselfmany episodes from the history of science have been retold in many, often incompatible ways, and a plurality of accounts need not be a sign of fundamental methodological problems. Plurality can be due to different sources; sometimes new sources are discovered; the same episode can be presented from different vantage points, and approached with different questions; new scientific knowledge can deepen our understanding of an episode, making previous accounts obsolete. This will lead to different accounts of the same historical episode in a natural and unsurprising manner.

The present case is different. The two competing views of the episode exhibit a considerable unity in perspective, methods, and sources. The Einstein scholars defending the diverging views worked over a period of ten years on a reconstruction and interpretation of one of the most important sources of Einstein scholarship, the "Zurich notebook". ${ }^{2}$ This long and close collaboration - I will call it the "genesis collaboration" - resulted in a jointly authored book, the four-volume opus magnum on the genesis of GR, Renn (2007). Despite the close collaboration, diverging views of crucial turning points of the genesis of GR have emerged. A contribution by John Norton defends a "minority view" of the episode in question, while the rest of the genesis collaboration, notably Jürgen Renn, Tilman Sauer and Michel Janssen defend what I will call the "majority view".

What is the disagreement? Einstein rejected a certain theory in the Zurich notebook in 1912-13, while the very same theory was reinstated for

\footnotetext{
${ }^{1}$ Most articles relevant to the present paper can be found in Janssen et al. (2007a,b).

${ }^{2}$ See the introduction in Janssen et al. (2007a) for remarks on the collaboration between Jürgen Renn, Tilman Sauer, Michel Janssen, John D. Norton, and John Stachel.
} 
a short period of time in the November of 1915. Why did Einstein reject the theory in the notebook, and why did he change his mind in November 1915? The majority claims that there is one major difference between the first and the second period, Einstein's awareness of so-called "coordinate conditions". This is a now-standard mathematical procedure for bringing the field equations of GR into correspondence with Newtonian gravitational theory. The majority argues that Einstein was unaware of coordinate conditions at the time of the notebook, and that he relied on so-called "coordinate restrictions", which severely limited the generality of the field equations and made the theory unacceptable. Only when he became aware of coordinate conditions did the theory become acceptable again. Norton finds this story implausible. He claims that Einstein must have had the modern notion of coordinate condition all along, but that he committed a different, more elaborate mistake. Importantly, this is a mistake that Einstein would repeat in the context of the famous "hole argument", a major roadblock on the path to the final theory of GR.

I will discuss methodological issues that arise from this non-trivial disagreement. Is this a dispute that cannot be settled despite a unity of evidence and methods? There is hope, I will argue, that we can dissolve the dispute. We can do better in the interpretation of the available evidence, in the reconstruction of the scientific and mathematical context of Einstein's struggle, and we can challenge the internal consistency of the two views. However, there are also fundamental methodological problems that we have to navigate. There are boundary conditions of rationality that enter into the reconstruction of historical episodes, for which there is no clear-cut justification.

I provide a short introduction to the history of GR and to the most important concepts in the upcoming section. I have tried to make the technical subject-matter of the episode accessible to non-specialists as much as possible. I then give an account of the two views, and of the reactions to the views by the respective opponents. After reviewing the arguments, I will probe the two views for weaknesses, and try to determine how we might settle the disagreement based on the available evidence, and on other considerations. Finally, I will discuss emerging methodological issues based on the previous discussion, and on methodological remarks by the parties involved.

\section{A Bird's Eye View of the Episode}

The story of the genesis of GR can be told in the form of a drama in three acts. $^{3}$ The main character is Einstein, with appearances by other famous physicists and mathematicians, notably his friend Marcel Grossmann, as well

\footnotetext{
${ }^{3}$ John Stachel (2007) has given an account of the genesis of GR in this form. The present section serves as an introduction; technical details are mostly relegated to footnotes.
} 
as various mathematical and physical theories and concepts. The premise of the drama is that not all is well in the house of gravitational physics. There is tension between the new theory of special relativity (SR), which constrains all physical theories and has a built-in finite speed of light, and the old Newtonian gravitational theory (NGT), which works by instantaneous action-at-a-distance. NGT will have to change, but how?

Enter Einstein, who sets out to reconcile the two theories by formulating a relativistic theory of gravitation. The core piece of the new theory will be a field equation that generalizes the gravitational Poisson equation. A gravitational field equation tells us how gravitation and matter, energy, and momentum hang together. The first two acts of the drama go relatively smoothly. In the first act, Einstein formulates the equivalence principle, which establishes a connection between accelerated reference frames and gravitational fields. In the second act, he finds that the best way to represent the gravitational potential in the field equation is by the metric tensor, a generalization of distance in Euclidean space to distance in space-times with variable curvature.

At the beginning of act three, Einstein has learned how to represent distance in space-time by the metric tensor, and he knows that energymomentum can be represented using the energy-momentum tensor. ${ }^{4}$ All that is left to do is to find the gravitational field equation, which tells us how space-time is influenced by the distribution of matter, energy, and momentum. Mathematically, the missing element of the field equation is a differential operator, which acts on the metric and thereby tells us how the metric and the energy-momentum tensor hang together. An appropriate differential operator generalizes the Laplace operator of the Poisson equation. This is where the reversal of fortune sets in. Einstein tests various candidates, straightforward generalizations of the Laplace operator, and also other candidates. However, none of them fits the bill. In a state of desperation, Einstein turns to Marcel Grossmann, his mathematician friend, for help.

Grossmann is indeed able to help. ${ }^{5}$ He finds a mathematical theory, the "absolute differential calculus", proposed by the Italian mathematicians Gregorio Ricci-Curbastro and Tullio Levi-Civita; this calculus is a framework that provides candidate differential operators for the field equations. The candidates are generally covariant, i.e., they keep their form under arbitrary coordinate transformations. The single most important object is the Riemann tensor; every possible generally covariant differential operator can be

\footnotetext{
${ }^{4} \mathrm{~A}$ detailed account of how the third act unfolded can be found in Renn and Sauer (2007)

${ }^{5}$ The collaboration between Einstein and Grossmann resulted in several publications, most importantly the so-called "Entwurf" ("outline") theory (Einstein and Grossmann, 1995), which contains the first detailed exposition of tensor calculus in the context of GR. The Entwurf theory does not yet formulate the final, correct field equations of GR; see Sauer (2014) for an account of Grossmann's contribution to GR.
} 
constructed from it. At the end of the drama, in November 1915, it will turn out that the absolute differential calculus and gravitational theory were right for each other all along. However, in 1912, Einstein and Grossmann do not realize this and the final, correct field equations have to wait behind the scenes.

Einstein and Grossmann use the Riemann tensor to derive the so-called Ricci tensor, a promising candidate. ${ }^{6}$ However, Einstein soon rejects the Ricci tensor as unsuitable for the field equation. This is a mistake, because the reasons for the rejection are ill conceived. The root of the problem has to do with the correspondence principle: The new field equation has to be put in correspondence with the old, classical gravitational field equationthe classical case should be recovered as a limiting case of the new, general theory. In order to do this, one has to consider other, intermediate cases, such as weak gravitational fields. At this point, Einstein is already experienced in handling such intermediate cases, but this experience does not serve him well: It generates wrong expectations about the form that special cases should take. The rejection of the Ricci tensor is a consequence of these wrong expectations.

Einstein then turns to the so-called "November tensor", a little brother of the Ricci tensor. The November tensor can be found by decomposing the Ricci tensor into two summands - one of these is the November tensor. It is not a generally covariant object, but its covariance group still includes some accelerated reference frames. At this point, the drama gets confusing. Einstein is able to show that the November tensor does not run into the same difficulties that had led him to eliminate the Ricci tensor. Despite this apparent progress, Einstein eliminates the November tensor as well, and it is not considered any further in the Zurich notebook. What prevented Einstein from investigating the November tensor further? What is more, years later, in November 1915, he returned to the November tensor and used it to formulate a version of general relativity. What made the November tensor acceptable again in November 1915? In later recollections, Einstein stated reasons for rejecting the November tensor. However, Einstein's explanations are not entirely satisfactory; his later recollections cannot fully resolve the puzzle.

Up to this point, the genesis collaboration agrees on how the drama unfolded, but now, the views start to diverge. The disagreement concerns the

\footnotetext{
${ }^{6}$ From here on, the story can only be reconstructed on the basis of the Zurich notebook. This part of the drama is now well understood thanks to the genesis collaboration; see Janssen et al. (2007a,b). The following account of Einstein's struggle is based on Norton (2007, sec. 1)

${ }^{7}$ The name was coined by the genesis collaboration; the November tensor became prominent in November 1915. It first appears on p. 22R of the Zurich notebook; see Fig. 1. I use the standard pagination; see Janssen et al. (2007a); Klein et al. (1995) for a facsimile of the notebook and Janssen et al. (2007b) for the commentary. Note that a facsimile of the Zurich notebook is also available online at Einstein Archive Online.
} 


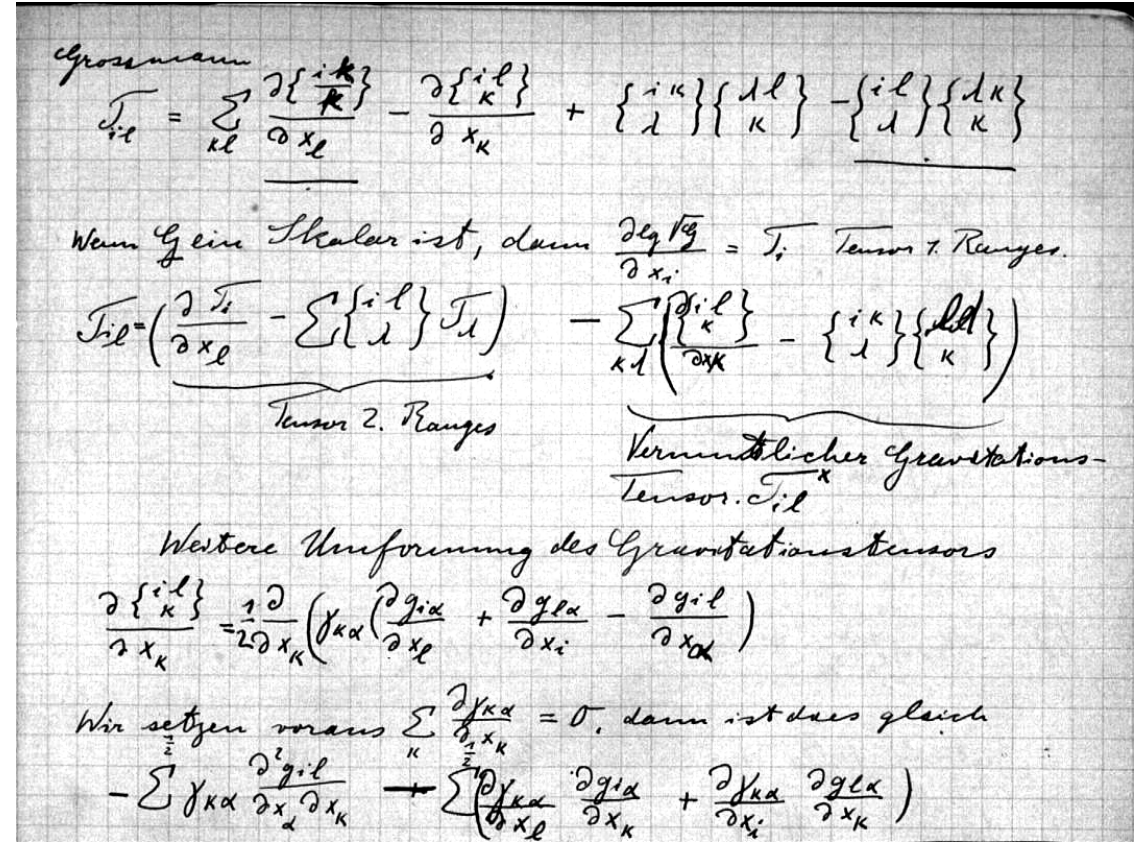

Figure 1: Top portion of page 22R of Einstein's Zurich notebook. The first line shows the Ricci tensor $\mathcal{T}_{i l}$, next to Grossmann's name. The Ricci tensor is then split up into two parts; the second part, labelled "Vermutlicher Gravitationstensor", is the so-called "November tensor." Albert Einstein Archives AEA 3-006. (C)The Hebrew University, Jerusalem. 
reason why exactly the November tensor was rejected in the Zurich notebook. If we compare Einstein's calculations involving the November tensor in the Zurich notebook in 1912-13, and in November 1915, there is one important difference. In the notebook, Einstein uses coordinate conditions in a way that differs from the modern usage. Coordinate conditions are a standard operation to recover the old gravitational theory from the new field equations. However, some of Einstein's calculations do not make sense from a modern point of view. Did Einstein deliberately apply coordinate conditions in a way that deviates from modern usage, or was he not aware of the modern usage? The two views under discussion disagree about how this question should be answered. The majority claims that Einstein did not have the modern notion of coordinate conditions at the time of the Zurich notebook. Norton, on the other hand, claims that Einstein was aware of coordinate conditions. Consequently, we get two different accounts of what led Einstein to reject the November tensor in the notebook.

\section{The Two Views}

We now turn to the two competing explanations of what went on in the November episode. The account given here is based on the detailed exposition in Norton (2007). ${ }^{8}$ Norton first presents the majority view, and then his own account. The reason why I use Norton's account is that it explicitly discusses, and accentuates, the contrast between the two views, while the other contributions do not focus on this disagreement. I will later turn to reactions of the majority view at the end of this section; this would serve as a sufficient corrective if Norton's account of the majority view were biased.

\subsection{The Majority View}

The majority view is that Einstein took field equations to have a special, weak field form not just in some particular coordinate system, but in general. This implies that Einstein had to reject the November tensor, as it does not have the required form. In order to understand this explanation, two different ways of using coordinate systems have to be distinguished.

\section{Coordinate Conditions vs. Coordinate Restrictions}

A generally covariant theory holds for arbitrary coordinate systems. In order to apply the equations to concrete situations, one has to introduce special coordinate systems. Coordinates can be introduced in different ways. One possibility is to specify differential equations that the coordinates have to

\footnotetext{
${ }^{8}$ There is an accessible presentation of Norton's view in Norton (2005). See Janssen et al. (2007a, pp. 11), for a brief overview of the evolution of Einstein scholarship concerning this episode.
} 
satisfy. This will fix a coordinate system only up to coordinate transformations that leave the differential equations invariant. Einstein may have used coordinates in two different ways in the notebook.

Coordinate conditions in the modern sense are a standard procedure if one wants to recover, say, the Newtonian limit. Newtonian gravitation in its standard formulation is not generally covariant, only covariant under Galilean transformations. Therefore, one may impose conditions on field equations of broader covariance if one wants to recover the Newtonian limit. Coordinate conditions do not restrict the covariance of the field equations; they are only used in the context of obtaining the Newtonian limit, e.g., when the imposition of a weak field assumption is not sufficient to recover Galilean covariance.

The examination of the Zurich notebook reveals that Einstein used coordinates in a second, non-standard way. The genesis collaboration has called this non-standard use coordinate restrictions. The introduction of coordinates into field equations always yields a new expression with restricted covariance. If coordinates are introduced as coordinate restrictions, the resulting expression is interpreted as the new field equation, which is not only valid under particular circumstances, but taken to be the gravitational field equation as such. The field equations before the application of coordinate restrictions are just an intermediate step in the derivation of the real field equations.

\section{Coordinate Restrictions for the November Tensor}

Einstein shows on p. $22 \mathrm{R}$ that if the so-called "Hertz condition" ${ }^{\prime}$ is applied to the November tensor, it reduces to the expected weak field form, an important intermediate step to the Newtonian limit. However, Einstein did not use the Hertz condition on p. $22 \mathrm{R}$ as a coordinate condition, but as a coordinate restriction. This can be seen by examining a calculation on p. 22L. Here, Einstein writes down two conditions, the Hertz condition, and the condition for "unimodular transformations"10, the covariance group of the November tensor. He then calculates the covariance of the Hertz condition under unimodular transformations, i.e., he determines the covariance group of the November tensor combined with the Hertz condition. This calculation does not make sense if he wants to use the Hertz condition as a coordinate condition. ${ }^{11}$ Subsequently, Einstein discards the Hertz condition. There is

\footnotetext{
${ }^{9}$ The name was coined by the genesis collaboration. It figures prominently in correspondence between Einstein and Paul Hertz; see, e.g., Renn and Sauer (2007, p. 184).

${ }^{10}$ Unimodular coordinates require that the determinant of the Jacobian of the coordinate differentials are equal to one.

${ }^{11}$ Only Galilean covariance is needed for the Newtonian limit, but it is easy to get Galilean covariance, as the condition is invariant under linear transformations, which implies Galilean covariance. However, Einstein does not eliminate terms from his calculation that would vanish under linear transformations. Therefore, he is not after linear transfor-
} 
a second instance in the Zurich notebook where Einstein used coordinate restrictions, not coordinate conditions.

\section{The Majority's Explanation}

According to the majority view, the concept of coordinate restriction explains the difference between the situation in the Zurich notebook and in November 1915 as follows. The November tensor does not have the form required for the Newtonian limit. If Einstein was unaware of the possibility of using coordinate conditions, this means that the November tensor was unacceptable as a candidate for the field equations, but it could still be used as an intermediary for a candidate with restricted covariance. Einstein therefore used the Hertz condition as a coordinate restriction; this produced a new candidate gravitation tensor with restricted covariance. It is documented that in November 1915, Einstein had acquired the notion of coordinate condition, and the November tensor became acceptable.

The majority and Norton agree that in the Zurich notebook, Einstein used coordinates in a way that can only be interpreted in terms of coordinate restrictions. Both views presuppose that Einstein did not use the Hertz condition as a coordinate condition. However, the majority view also assumes that Einstein was unaware of the possibility of interpreting the application of the Hertz condition to the November tensor as a coordinate condition. It is on this point that the majority and Norton disagree.

\subsection{Norton on the Majority View}

The majority assumes that Einstein was unaware of coordinate conditions. How plausible is this assumption? According to Norton, this question cannot be settled on the basis of the available evidence. There are instances where Einstein used coordinate restrictions in the notebook. However, in other cases, it is unclear how he interpreted the use of coordinates; the "harmonic coordinates" 12 are an example for the latter. Einstein did not check the covariance group of the harmonic coordinates. Norton concludes that nothing in the notebook precluded Einstein from being aware of coordinate conditions.

\section{"Vermutlicher Gravitationstensor"}

One central piece of evidence speaking against the assumption that Einstein was unaware of coordinate conditions can be found on p. 22R of the notebook; see Fig. 1. Einstein splits the Ricci tensor into two parts, one of which is the

${ }^{12}$ Einstein used harmonic coordinates to recover the weak field form of the metric in the context of the Ricci tensor. Harmonic coordinates were available in the mathematical literature as "isothermal coordinates" at the time of the notebook.
} 
November tensor. He marks the November tensor with the label "presumed gravitation tensor" ("Vermutlicher Gravitationstensor"). Norton writes that, at this point, it must have been clear to Einstein that the November does not have the necessary form to reduce to the Newtonian limit without a further condition; he was sufficiently experienced to see this immediately. But if this is the case, the label would be inappropriate. The November tensor would not be the "presumed gravitation tensor", but just another intermediate step on the way to the right candidate; it would play the same role as the Ricci tensor.

Now, this could be an oversight on Einstein's part; he could have assigned the label in haste. However, according to Norton, this is implausible. For one, the page is neatly written, more like a summary than a hasty calculation. Also, Einstein had probably discussed the November tensor with Grossmann at this point - Grossmann's name appears on the page. Furthermore, the Hertz condition also appears on this page. Einstein's hope may have been that the November tensor reduces to the right Newtonian limit with the help of the Hertz condition. However, if he interpreted the Hertz condition as a coordinate restriction, then the November tensor would not be the "presumed gravitation tensor", but just an intermediary.

\section{Evidence Against Coordinate Conditions?}

Is there any clear evidence that Einstein was unaware of coordinate conditions at the time of writing the notebook? Norton argues that this is not the case. First, Einstein did not mention problems with coordinate conditions later on. This is relevant because he frequently commented on mistakes committed during the genesis of GR, from the hole argument to the wrong assumption that the static metric has to be spatially flat. However, he never mentioned problems with coordinate conditions later on. Second, coordinate conditions are not needed in order to recover the Newtonian limit of the Entwurf; this explains why he never mentioned problems with coordinate conditions in this context. However, he also failed to mention them in other contexts where they may have been relevant. Third, Einstein had shown that he was aware of different ways of using coordinates. A lack of awareness of how to use coordinate conditions is implausible, because coordinates and their use was one of Einstein's motivations for the construction of a generalized theory of relativity in the first place.

According to Norton, all of this suggests, or at least leaves open the possibility, that Einstein was aware of the possibility of using coordinate conditions in the notebook. Could Einstein have considered both coordinate conditions and coordinate restrictions at the same time? If Einstein was aware of coordinate conditions, new puzzles have to be solved. It is the purpose of Norton's account to spell out how the events surrounding the November tensor unfolded if Einstein was aware of coordinate conditions. 


\subsection{Norton's View}

Two problems have to be solved if we assume that Einstein was aware of coordinate conditions at the time of the notebook. First, we have to explain why Einstein was unable to recover the Newtonian limit of the November tensor using the Hertz condition, because this calculation features on p. 22R. Second, we have to explain why he stopped using coordinate conditions in combination with the November tensor in the notebook. Of course, not any explanation will do. If coordinate restrictions are not the explanation, Einstein must have made some other mistake. It would be easy to just invent an additional error - but this is not sufficient, as Norton points out: "The real difficulty is to establish that the error was really committed" (Norton, 2007, p. 748).

There is one misconception that satisfies these requirements. Einstein explicitly defended this misconception, and admitted later on that it had been a mistake. It is the mistake of "attributing an independent reality to coordinate systems"13, which was later central in the infamous hole argument. This mistake might also explain the puzzles of the November episode.

Norton conjectures that this misconception already shows up in the notebook. This would explain why Einstein gave up on the use of coordinate conditions on p. 22R of the notebook, and why he accepted the restricted covariance of the Entwurf equations. He only reversed his mistake in November 1915. If Einstein made this mistake in the context of the November tensor, the result would be that if a coordinate condition is applied, the covariance of the theory is reduced, and coordinate conditions are not only valid in the context of the Newtonian limit, but in general. Einstein may have realized this on p. 22R, and therefore abandoned the use of the Hertz condition as a coordinate condition.

Norton's conjecture presupposes that the mistake of attributing an independent reality to coordinates was tacit. The mistake was only made fully explicit when Einstein withdrew the hole argument in late 1915. If he had realized that he attributed an independent reality to coordinates in the notebook, he would not have endorsed the position. It is plausible that Einstein was not clear on this point, as he later had problems to spell out what exactly had gone wrong in the hole argument.

\section{The Hole Argument}

Norton's conjecture is based on an analogy to the hole argument. Einstein came up with the hole argument in late 1913. It served as an argument against the requirement that the field equations of general relativity should

\footnotetext{
${ }^{13}$ This formulation is used in Norton (2007) for this particular misconception. I will use it as a technical notion in the present paper. It does not apply to the mistake of, say, using coordinate restrictions instead of coordinate conditions.
} 
be generally covariant.

The argument runs as follows. ${ }^{14}$ Take a region of space-time that is free of matter. In this region, the only field that can make a physical difference is the metric. The metric $g$ is a function of the coordinate system $x$, written $g(x)$. Now, we can choose different coordinates $x^{\prime}$, which agree with $x$ everywhere except in the region free of matter, where they deviate smoothly from $x$. The transformation from $x$ to $x^{\prime}$ will yield a different representation of the metric, written $g^{\prime}\left(x^{\prime}\right)$. Einstein interpreted $g^{\prime}\left(x^{\prime}\right)$ to be the same field as $g(x)$, written in different coordinates - one and the same metric has different components in different coordinate systems; this does not constitute a physical difference. If we accept general covariance, we can also write the metric $g^{\prime}$ in terms of the original coordinate system $x$. However, this gives rise to the problem that the new metric in terms of the original coordinates, $g^{\prime}(x)$, deviates from the $g(x)$ inside the designated space-time region: it yields a different field, despite being expressed in the same coordinates. This constitutes a physical indeterminacy, which is unacceptable; the culprit is general covariance, which, has to be rejected.

The hole argument is defective, as is well known. The mistake is to attribute a different physical meaning to the two solutions $g(x)$ and $g^{\prime}(x)$. They are just mathematically different expressions of the same physical field. The source of the mistake is to (implicitly) attribute an independent reality to the coordinate system $x$. If the coordinate system would pick out spacetime points uniquely and independently of $g$ and $g^{\prime}$, then a disagreement of $g$ and $g^{\prime}$ would be due to physical properties of the space-time point. However, space-time points are only individuated in virtue of the metric field. The difference is mathematical, not physical.

\section{Norton's Conjecture}

Norton's conjecture is that Einstein made the same mistake in the case of the November tensor: he attributed an independent reality to coordinates. As a consequence, the covariance of the theory was restricted to the coordinate conditions used to recover the Newtonian limit.

Einstein's reasoning might have proceeded along the following lines. The November tensor, while not generally covariant, is covariant under unimodular transformations. In the notebook, Einstein examined the transformation from Minkowski coordinates, $x_{\mathrm{SR}}$, to uniformly rotating coordinates, $x_{\mathrm{ROT}}$. This transformation is nothing but a change of coordinates. It yields a different expression for the metric: Starting from the Minkowski metric $g^{\mathrm{SR}}\left(x_{\mathrm{SR}}\right)$, one arrives at a different metric $g^{\mathrm{ROT}}\left(x_{\mathrm{ROT}}\right)$ in rotating coordinates. Given that the Minkowski metric is a solution of the November theory, and because uniformly rotating coordinates are unimodular, $g^{\mathrm{ROT}}\left(x_{\mathrm{ROT}}\right)$ is also a solution of the November theory. This is not yet problematic.

\footnotetext{
${ }^{14}$ See Norton (2011) for a discussion of the hole argument.
} 
However, it is only unproblematic insofar as $g^{\mathrm{SR}}$ and $g^{\mathrm{ROT}}$ are solutions in different coordinate system. This is where Einstein might have made a mistake by interpreting the transformation to $g^{\text {ROT }}$ differently: He may have (implicitly) presupposed that $g^{\mathrm{SR}}$ and $g^{\mathrm{ROT}}$ both have to be solutions in the same coordinate system. He used the Hertz condition to bring the November tensor into the form required for the Newtonian limit. By checking whether both $g^{\mathrm{SR}}$ and $g^{\mathrm{ROT}}$ are compatible with the Hertz condition, he implicitly assumed that these two expressions needed to be compatible with the same coordinate system, namely the coordinates $x_{\text {HERTZ }}$ compatible with the Hertz condition. He thus expected that $g^{\mathrm{SR}}\left(x_{\text {HERTZ }}\right)$ and $g^{\mathrm{ROT}}\left(x_{\text {HERTZ }}\right)$ both have to be solutions to obtain the Newtonian limit if one uses the Hertz condition. This, however, is impossible, because the Hertz condition is not compatible with $g^{\mathrm{ROT}}$. This may have led to the rejection of the November tensor.

The problem is that Einstein used the Hertz condition to check compatibility of different expressions of the metric. This effectively limited the covariance of the theory to the covariance of the coordinate conditions of the Newtonian limit - the covariance of the Hertz condition in the present case - and there is no longer a difference between coordinate conditions and coordinate restrictions. Consequently, at this point, Einstein turned to using coordinate restrictions, as they had the advantage of yielding simplified field equations. This also explains why Einstein checked the covariance of the Hertz condition - he simply attributed a physical meaning to this condition.

\section{Evidence for Norton's Conjecture}

There is no direct evidence for Norton's conjecture in the Zurich notebook or in the Entwurf, i.e., it is unclear whether Einstein attributed an independent reality to coordinate systems at this time. We only have evidence that he did so in the context of the hole argument. Norton finds that the conjecture is compatible with Einstein's pronouncements on coordinate systems between 1912 and 1915 and with his attitude towards general covariance. One piece of evidence speaking in favor of Norton's conjecture is a letter to de Sitter, in which Einstein ties the lack of rotational covariance of the Entwurf field equations to the rejection of the hole argument. The conjecture establishes a direct connection between the hole argument and rotational covariance.

Norton locates the strength of the conjecture in its explanatory power, under the assumption that Einstein was aware of coordinate conditions - we have seen Norton's reasons for assuming that Einstein was aware of coordinate conditions in section 3.2 above. The conjecture explains why Einstein gave up on using coordinate conditions in the notebook, it explains why he later thought that he did not succeed in deriving the Newtonian limit from the November tensor, despite a calculation that appears to show the contrary, and it explains his indifference towards general covariance in the 
Entwurf phase prior to the hole argument.

\subsection{The Majority on Norton's View}

The majority fraction of the genesis collaboration has not reacted to Norton's conjecture in detail. The majority maintains that the distinction between coordinate conditions and coordinate restrictions was sufficient for Einstein's rejection of the November tensor in the notebook, and they attribute the revival of the November tensor in 1915 to Einstein's realization that he could use coordinate conditions. Here are two reactions of the majority to Norton's conjecture.

\section{Jürgen Renn}

Jürgen Renn (2004, sec. 2) discusses the question as to why Einstein abandoned promising differential operators, such as the Ricci and the November tensor, in the notebook. Renn asks whether Einstein may have been unaware of coordinate conditions in the Zurich notebook - is it possible that "Einstein could have been guilty of such a trivial error?" (Ibid., p. 12). He points out that Einstein used harmonic coordinates in the notebook in the context of the Ricci tensor, which suggests that Einstein was aware of coordinate conditions. The calculations surrounding the November tensor, however, tell a different story; they show that Einstein's understanding of coordinate conditions differs from the modern view. In particular, Einstein checked the transformation group of coordinate conditions, which does not make sense according to the modern view; see section 3.1 above. What could have induced Einstein to think that coordinates impose real restrictions on the field equations?

Renn briefly discusses Norton's answer to this question. He characterizes Norton's account as attributing a deep and "conceptual, if not metaphysical" (p. 14) error to Einstein. Renn is not convinced by Norton's account:

The evidence available makes it, in my view, implausible that this was indeed Einstein, Äôs pitfall in early 1913. If he committed an error conceptually close to the hole argument then it becomes incomprehensible why, as the historical documents indicate, Einstein only formulated this argument as late as summer 1913, and from then on regarded it as the life belt of the 'Entwurf' theory, while, before that, he considered its lack of being generally covariant as a shameful dark spot. (Ibid., p. 14)

Renn mentions the Besso memo in support of this claim. The Besso memo, probably written on the 28th of August 1913, contains a preliminary version of the hole argument. ${ }^{15}$ Renn concludes that the hole argument, or

\footnotetext{
${ }^{15}$ The argument for dating the Besso memo is given in Janssen (2007).
} 
the reasoning underlying the hole argument, is not the "original sin" leading to the abandonment of the November tensor, and that the only viable explanation is that Einstein really did not have the modern notion of coordinate conditions.

\section{Michel Janssen}

Michel Janssen (2007) comments on Norton's argument in the context of the so-called "Besso Memo", which contains early traces of the hole argument, as well as reasons for rejecting it. Janssen writes: "It is my belief that Einstein used coordinate restrictions in the Zurich Notebook simply because he did not yet have the modern understanding of coordinate conditions. No further explanation is needed. Consequently, I am skeptical about Norton, Äôs conjecture" (Ibid., p. 828). Janssen does not elaborate on why he believes that Einstein did not yet have the modern notion of coordinate conditions, and he does not give further arguments against Norton's conjecture.

In sum, the majority is skeptical of Norton's solution, but there is no sustained engagement with Norton's arguments. Both Renn and Janssen point out that the hole argument, which is in the background of Norton's conjecture, has a philosophical, conceptual, or even metaphysical ring to it. This might indicate that Renn and Janssen consider Norton's proposal to be somewhat speculative.

\section{How to Resolve the Disagreement}

Can the dispute between the majority and Norton be resolved? I agree with Norton that there is, at present, no evidence that could definitively settle the issue. However, I am optimistic that progress can be made. In this section, I will suggest several ways in which the debate can be brought forward. This will prepare the ground for the discussion of the more fundamental methodological issues in the next section.

Here is a sketch of the disagreement. The main point of contention is whether Einstein was aware of coordinate conditions at the time of the notebook. The distinction between coordinate conditions and coordinate restriction, emphasized by the majority view, only explains Einstein's rejection of the November tensor if he was not aware of coordinate conditions. If it were possible to decide whether or not Einstein was aware of coordinate conditions, then the disagreement would simply disappear. I will reexamine this point in section 4.1. Norton claims that Einstein must have been aware of coordinate conditions. The central piece of evidence for this claim is the "presumed gravitation tensor"; this is the topic of section 4.2. If Einstein was aware of coordinate conditions, the change from coordinate conditions to coordinate restrictions is not accounted for by the distinction between coordinate conditions and restrictions, and a different explanation for the 
use of coordinate restrictions is needed. Now, Norton's conjecture comes into play. The occurrence of coordinate restrictions is explained by Einstein's mistake of attributing an independent reality to coordinate systems, a mistake he made in the context of the hole argument later on. By implicitly assigning independent reality to coordinates, Einstein collapsed the distinction between coordinate conditions and coordinate restrictions in the notebook. Norton comments on the (theoretical) virtues of his and the majority's explanation in various passages. This will be the topic of section 4.3 .

\subsection{Einstein's Knowledge of Coordinate Conditions}

Did Einstein have the notion of coordinate conditions at the time of the notebook? Both views seem to agree that, prior to November 1915, there is no instance where Einstein clearly used coordinate conditions in the modern sense. ${ }^{16}$ However, direct evidence is not all that matters. We also have to take into consideration in how far coordinate conditions were available in the literature at the time of the notebook. If the notion was available, and if only in part, the claim that Einstein was aware of coordinate conditions gains plausibility: he simply had to check the relevant literature.

Both the majority fraction and Norton mention this point only in passing. There is hardly any discussion of the relevant physical and mathematical literature. A point mentioned by both parties is that Einstein took harmonic coordinates from the mathematical literature. Harmonic coordinates were known as "isothermal coordinates" in the literature on differential geometry such as Bianchi (1910) and Wright (1908). We know that these works were familiar to Einstein. But how relevant is this particular kind of coordinates to the modern notion of coordinate conditions?

To answer this question, we have to examine the two sources just mentioned. Here is a brief recapitulation. In Bianchi (1910, ch. 3), a textbook on differential geometry, it is shown that we can find a coordinatization of a surface such that the line element takes a particularly simple form. Such a parametrization exists if the second Beltrami parameter vanishes; this is mentioned by Einstein on p. 19L, as Norton points out. Bianchi also discusses the geometrical significance of these systems of curves. Wright (1908) is a monograph on quadratic differential forms; "isothermal systems of curves" are discussed in the context of applications of invariant theory. Wright also states that isothermal systems are tied to the vanishing of the second Beltrami parameter. ${ }^{17}$ This shows that harmonic, or isothermal, coordinates were well understood mathematically.

\footnotetext{
${ }^{16}$ See Janssen and Renn (2007, sec. 1.5) for an argument to this effect. This argument is neutral with respect to the disagreement discussed here.

${ }^{17}$ Note that Ricci and Levi-Civita (1901) discuss isothermal surfaces.
} 
However, the mathematical notion of harmonic coordinates, and the notion of coordinate condition, are quite far apart. Most importantly, the mathematical literature considered above is completely silent on the issue of using coordinates in a physical context. ${ }^{18}$ There is no discussion of using coordinates to obtain, say, the Newtonian limit - this is not surprising; after all, these are works on differential geometry and invariant theory, not on physics. However, adapting coordinates to particular situations is the key ingredient of the modern notion of coordinate conditions. When considering the Newtonian limit, we can use a particular set of coordinates for our field equations, which does not affect the generality of the equations. This idea does not feature in the mathematical literature. It would be more fruitful to search the physical literature for seeds of the notion that Einstein needed.

What does this mean for the two diverging views? If the key ingredient to the modern notion of coordinate condition was not available in the literature, Einstein had to find the notion on his own. However, there are not many traces of this search. Thus, the story in Janssen and Renn (2007, sec. 1.5) that the modern notion was forced upon Einstein only in 1915 gains plausibility as an account of how Einstein did arrive at the modern notion. This speaks in favor of the majority view.

\subsection{Evidence Against the Majority View: "Vermutlicher Grav- itationstensor"}

Norton adduces one central piece of evidence against the majority view: the labeling of the November tensor as the "presumed gravitation tensor" ("Vermutlicher Gravitationstensor") on p. 22R of the notebook; see section 3.2 above. Norton argues that if we adopt a literal reading of this label, then the November tensor itself is the gravitation tensor, and not an intermediate step on the way to a different gravitation tensor. This implies that Einstein would not apply a coordinate restriction to the November tensor, but a coordinate condition.

How convincing is this argument? Unfortunately, there is no response by the majority, and the commentary on the notebook in Janssen et al. (2007b) does not further elaborate on the label. What are the possible explanations for labeling this part of the expression as the "presumed gravitation tensor"? We cannot reject Norton's explanation and still claim that the label is accurate. But other explanations for the label are possible.

Norton writes that the page is neatly written, indicating that p. $22 \mathrm{R}$ may have served as a summary of a calculation or a discussion. One alternative explanation is that the label served as a mnemonic device: Einstein simply wanted to mark this part of the expression as relevant, as opposed to its other

\footnotetext{
${ }^{18}$ Relevant parts of the modern notion may be discussed elsewhere in the mathematical literature. There are useful remarks on the history of "Euclidean geometry by means of general coordinates" in Veblen (1927, pp. 66).
} 
parts, and to be used as a basis for a "presumed gravitation tensor". On this account, the label would have a contrastive role instead of a descriptive one. Maybe it would have been tedious to write down that the expression should be the basis for the gravitation tensor, because it may have been clear at this point that this is out of the question. This does not refute Norton's explanation, but it show that other explanations of the label are possible.

A different way of deciding whether or not Norton's explanation is plausible is to consider other instances of labeling in the notebook. Here is an example. Einstein labels a different object as "vermutlicher Gravitationstensor" on p. 9L. ${ }^{19}$ The context of this second occurrence of a candidate gravitation tensor is different-the issue on p. 9L is the gravitational stress-energy tensor. Also, the handwriting on p. 9L is less tidy than on p. 22R. It is not clear whether the use of the label on p. 9L speaks in favor or against Norton's interpretation of the label on p. 22R, but discussing other instances of labeling in the notebook might convey a feeling for Einstein's usual practice.

\subsection{Theoretical Virtues: Simplicity and Explanatory Power}

An issue that is repeatedly discussed by Norton is the simplicity of the two views. He thinks that the majority view is the simpler account of why Einstein considered the November tensor to be untenable in the notebook. The majority view can account for Einstein's actions in virtue of just one distinction, that between coordinate conditions and coordinate restrictions, and there is no need to explain why Einstein used coordinate conditions in some contexts, and not in others. Norton, on the other hand, tells a more complex story, involving a mistake that was made twice, but only appeared in writing once, in the context of the hole argument.

Of course, Norton's view is more complex for a reason - it assumes that Einstein was aware of coordinate conditions, and the view is therefore able to account for the available evidence under this assumption. Much hinges on this starting point: Norton's view rejects the premise of the majority view; this necessitates the introduction of a more complicated explanation. If the assumption of Einstein's awareness of coordinate conditions holds water, then the complexity of Norton's view does not speak against it.

Norton also discusses a second notion of simplicity, a quantitative parsimony of mistakes. On this notion of simplicity, Norton's view is simpler than the majority view, because it does not "multiply mistakes beyond necessity". The majority view has to attribute an "elementary blunder" to Einstein, that of not being aware of coordinate conditions. On Norton's view, Einstein committed the elaborate mistake of attributing an independent reality to coordinate systems in the context of the November tensor, and on top

\footnotetext{
${ }^{19}$ Note that the two labels are identical in German (up to the capital letter), but translated differently in the commentary; see Janssen et al. (2007b, p. 555 and p. 647).
} 
of this, Einstein repeated this very mistake in the context of the hole argument. Norton thus proposes a kind of "common cause explanation": Both the rejection of the November tensor, and the hole argument, are due to the same kind of blunder.

How convincing is this "common cause explanation"? One way to criticize it is along the lines of Jürgen Renn (2004); see section 3.4: Under the assumption that the mistake was already at work in the notebook, Renn contends, it should have had other observable effects, which, however, we do not find. This is a problem of (unobserved) consequences of the mistake conjectured by Norton.

A different line of criticism is to call into question that we are in fact dealing with just one mistake. One problem might be that the mistake is not exactly the same in the case of the November tensor and in the case of the hole argument; the two situations are only analogous. However, if it is not exactly the same mistake in both cases, should we still count it as one mistake? Norton emphasizes that the analogy is quite strong. There is not only a qualitative, but a formal parallel between the two mistakes, as we have seen in section 3.3. If the parallel between the two situations really is that strong, the claim of quantitative parsimony seems legitimate.

This observation brings a different problem of Norton's common cause explanation to the fore. His view is based on a sophisticated Einstein, who does not commit elementary blunders, especially when it comes to coordinate systems. Norton therefore has to presuppose that the mistake of attributing an independent reality to coordinates was implicit - if Einstein had been completely aware of the ramifications of the mistake, he would not have made it. However, if the erroneous reasoning is only implicit, the tie to Einstein's later mistake in the hole argument gets weaker. We appreciate the link between the two mistakes because the parallel is made explicit. If Einstein would have seen the analogy between the mistakes as presented by Norton, and given Einstein's competence when it comes to coordinates systems - would he still have embraced it? If the parallel between the two occasions of the mistake was clear to Einstein, it is less plausible that Einstein would have made the mistake. If, on the other hand, the parallel is unclear, the common cause explanation gets weaker, and it is dubious that just one mistake was committed. There is a tension between Einstein's committing the mistake consciously, and the force of the common cause explanation.

\section{Methodological Issues}

In the previous section, I suggested how we might settle the disagreement in substance. Now I will take a step back and reflect on the nature of the disagreement. Can we settle the dispute on the basis of the available evidence? Is the disagreement a matter of personal taste, or are the two 
views based on fundamental methodological differences?

The nice thing about the present case is that the disagreement arose within a group that has worked in close collaboration on the same evidence, shares a large part of historical methodology, and has tried to come up with a common interpretation of the evidence. The dispute is not rooted in the fact that the two views take different sources into account; rather, the disagreement concerns the interpretation of evidence. This makes the problem of deciding between the two views hard, and interesting.

Norton, and the majority fraction of the genesis collaboration, have repeatedly commented on their methodology. These methodological remarks will be my starting point. I will focus on two lessons we can learn from the present case. The first lesson is positive: The dispute is not a mere matter of taste - progress is possible. The second lesson is more guarded; it points out a fundamental methodological problem, the question as to how much rationality we should ascribe to a historical actor.

\subsection{Reconstructing Einstein}

In the introduction of his paper, Norton characterizes the problem of deciding between the two views as a puzzle that lies on the boundary between the clear and the obscure: It is possible to formulate candidate solutions, but there is not enough evidence to reach a final verdict. What is at stake is not the evidence, but the interpretation of evidence, and theoretical considerations. Norton suggests that we should evaluate the plausibility of the different views. He writes that when we invoke plausibility, "our personal Einsteins speak as much as evidence" (Norton, 2007, p. 745).

Is a "personal Einstein" really that important in Norton's analysis? On closer inspection, it is not. Norton certainly assigns weights to evidence in a different way than the majority, but he is always careful to defend these weights. Arguments decide the outcome of the dispute, not subjective factors. Instead of different "personal Einsteins", I prefer to think of different, argued reconstructions of Einstein that are subject to critical evaluation. These arguments can be probed further in order to advance the discussionwe can push the boundaries of the unknown. Above, we saw three ways of probing the reconstructions of Einstein.

First, if direct evidence cannot settle an issue, we may take further sources and background knowledge into account. Did Einstein know about coordinate conditions at the time of the notebook, or did he not? I have suggested in section 4.1 that we may look at the context if we cannot decide this question on the basis of the notebook. We can try to determine how likely it would have been to know about coordinate conditions, given the mathematical and physical background. If the notion of coordinate condition was not available at the time, and if there is no evidence of how Einstein acquired the notion, it gets more implausible that he had the modern notion. 
Second, once we have proposed a certain reconstruction, it can be scrutinized anew on the basis of the available evidence. One example is the labeling of the "presumed gravitation tensor"; see section 4.2 above. Norton's argument is based on the assumption that Einstein was accurate when it comes to labeling. We can now revisit the notebook and compare this instance of labeling with other instances, and thereby decide whether Einstein really is that accurate, or whether we can come up with an alternative explanation that trumps Einstein's accuracy. A second example of reevaluating a given reconstruction is Renn's point that the mistake of attributing an independent reality to coordinates should have had observable consequences before the hole argument.

Third, we can check the internal consistency, or plausibility, of the reconstructions. I argued in section 4.3 that there is an tension in Norton's account between the quantitative parsimony of mistakes on the one hand, and how consciously the mistake was made on the other.

\subsection{Historical Errors: A Dilemma}

The episode under dispute depends on the attribution of mistakes to the historical actor, Einstein. This generates methodological problems. Norton formulates one of these problems as follows: "Of course it is always possible to invent hidden errors varying from the trivial slip to the profound confusion, tailor made to fit this or that aberration" (Norton, 2007, p. 748). If we are interested in explaining the actual course of events in a historical episode, the indiscriminate introduction of errors threatens to trivialize the account.

Norton argues that this problem can be overcome by a quantitative parsimony of mistakes. His account of the episode satisfies this constraint: "What is appealing about the conjecture is that it requires us to posit no new errors" (Ibid., p. 781). According to Norton's conjecture, we do not have to multiply mistakes beyond necessity, because Einstein repeated the mistake of attributing an independent reality to coordinate systems in the context of the hole argument. I have already pointed out a material problem of Norton's conjecture in section 4.3: the mistake might not be exactly the same in both situations, but only analogous.

However, there is an even more fundamental problem lurking in the background. Why should we attribute as few errors as possible to Einstein in principle? Isn't it natural that scientists commit mistakes? Isn't it problematic to presuppose that historical actors proceed in a quasi-rational manner? On the one hand, we can explain any historical episode if we presuppose the right kind of error at the right moment in history. On the other hand, do we have good reasons to minimize the amount of errors we conjecture in our historical accounts? It appears that we face a dilemma. The second horn of the dilemma has been forcefully formulated by Michel Janssen (2007, p. 832): 
So, to put it somewhat bluntly, whenever one encounters a passage containing what on the face of it looks like an error on Einstein's part, the strategy is to look for an interpretation in which the apparent error is the manifestation of some deep conceptual difficulty that had to be overcome before general relativity as we know it could be formulated.

The worry might be that we end up with a reconstruction of Einstein that is too rational in that it only deviates from the perfect path of discovery if Einstein encounters "deep" difficulties. Janssen discusses this issue in section 5 of his paper against the background of Einstein scholarship since the 1980s. Before Norton's and Stachel's groundbreaking interpretation of the notebook, historical reconstructions of the genesis of general relativity attributed trivial errors to Einstein. For example, these early accounts assumed that Einstein did not know that if one transforms the components of the metric using coordinate transformations, the resulting expression is not physically different from the untransformed metric. Norton and Stachel ruled out this possibility, thus avoiding the first horn of the dilemma. Janssen finds that while this was an improvement, Norton got too close to the second horn of the dilemma with his "excessively acute Einstein". Janssen prefers a different, "opportunistic" Einstein, who did not follow up on inconsistencies if they threatened his pet principles.

There is probably no silver bullet for this dilemma. We should avoid the implausible attribution of trivial errors to Einstein, but also steer clear of an overly charitable interpretation, or of "overly complex errors". ${ }^{20}$ We can only avoid these pitfalls by scrupulously reconstructing Einstein from the available evidence.

\section{Conclusion}

We have seen two accounts of the same historical episode. Both are based on the same evidence, and still they disagree. The reason for the disagreement lies, first, in the weight assigned to the evidence. For example, Norton emphasizes the case of the "presumed gravitation tensor"; the majority view does not discuss this point. A second source of disagreement is a different view on the (background) knowledge we may attribute to Einstein, or when and how Einstein acquired this knowledge. Did Einstein know about the freedom to apply coordinate conditions? Here, both camps have merely sketched the context. Third, the two views have, implicitly and explicitly,

\footnotetext{
${ }^{20}$ It would be desirable to get a better systematic understanding of the role of errors in this episode. Such an understanding might be gained on the basis of the so-called "dynamical inferential conception" of the application of mathematics, proposed in Räz and Sauer (2015). This framework systematizes different kinds of mistakes that can be made in the context of applying mathematics to empirical problems.
} 
emphasized different theoretical virtues. The majority emphasizes one distinction as crucial, while Norton has a more intricate story that connects the notebook to one other important episode in the genesis of GR. Norton's position might be more speculative and, therefore, also more susceptible to criticism.

The methodological discussion has shown that fundamental methodological issues play a role in the disagreement as well. On the one hand, the majority view attributes a mistake to Einstein that may seem elementary from a modern perspective. On the other hand, Norton's view constructs an elaborate mistake, which would persist for some time and resurface later. While the first view may run the risk of telling too simple a story and trivialize the episode, the other may be conceived as painting a picture of Einstein that is too rational.

Despite these difficulties, there is reason for hope; progress is possible at all points. We can try to decide on the relevance of evidence by comparing similar cases; we can make an effort to reconstruct the background knowledge; we can adduce philosophical and psychological theories in order to understand how the transfer of knowledge from one field to another works, and to understand what kind of mistake we may attribute to historical actors. All of this will lead to an improved reconstruction of Einstein.

\section{Acknowledgements}

I thank John Norton and Raphael Scholl for comments on previous drafts of the paper, and Tilman Sauer for comments and fruitful discussions concerning the genesis of GR.

\section{References}

Bianchi, L. 1910. Vorlesungen über Differentialgeometrie. Teubner, 2nd ed.

Einstein, A. and M. Grossmann. 1995. Entwurf einer verallgemeinerten Relativitätstheorie und einer Theorie der Gravitation. In Klein et al. (1995), pp. 302-43.

Janssen, M. 2007. What Did Einstein Know and When Did He Know It? A Besso Memo Dated August 1913. Vol. 2. Einstein's Zurich Notebook: Commentary and Essays of Janssen et al. (2007b), pp. 785-838.

Janssen, M., J. D. Norton, J. Renn, T. Sauer, and J. Stachel, eds. 2007a. The Genesis of General Relativity, vol. 1. Einstein's Zurich Notebook: Introduction and Source. Dordrecht: Springer.

- 2007b. The Genesis of General Relativity, vol. 2. Einstein's Zurich Notebook: Commentary and Essays. Dordrecht: Springer. 
Janssen, M. and J. Renn. 2007. Untying the Knot: How Einstein Found His Way Back to Field Equations Discarded in the Zurich Notebook. Vol. 2. Einstein's Zurich Notebook: Commentary and Essays of Janssen et al. (2007b), pp. 839-925.

Klein, M. J., A. J. Kox, J. Renn, and R. Schulmann, eds. 1995. The Collected Papers of Albert Einstein, vol. 4: The Swiss Years: Writings, 1912-1914. Princeton, New Jersey: Princeton University Press.

Norton, J. D. 2005. A Conjecture on Einstein, the Independent Reality of Spacetime Coordinate Systems an the Disaster of 1913. In A. J. Kox and J. Eisenstaedt, eds., The Universe of General Relativity, vol. 11 of Einstein Studies. Basel, Boston, Berlin: Birkhäuser, pp. $67-102$.

2007. What Was Einstein's "Fateful Prejudice"? Vol. 2. Einstein's Zurich Notebook: Commentary and Essays of Janssen et al. (2007b), pp. 715-83.

2011. The Hole Argument. Http://plato.stanford.edu/entries/spacetime-holearg/.

Räz, T. and T. Sauer. 2015. Outline of a dynamical inferential conception of the application of mathematics. Studies in History and Philosophy of Modern Physics 49: 57-72.

Renn, J. 2004. Standing on the Shoulders of a Dwarf: General Relativity: A Triumph of Einstein and Grossmann's Erroneous "Entwurf" Theory. In In the Shadow of the Relativity Revolution, Preprint 271. Berlin: Max Planck Institute for the History of Science, pp. 5-20.

Renn, J., ed. 2007. The Genesis of General Relativity (4 vols.). Dordrecht: Springer.

Renn, J. and T. Sauer. 2007. Pathways out of Classical Physics. Einstein's Double Strategy in his Search for the Gravitational Field Equation. Vol. 1. Einstein's Zurich Notebook: Introduction and Source of Janssen et al. (2007a), pp. 113-312.

Ricci, M. and T. Levi-Civita. 1901. Méthodes de calcul différentiel absolu et leurs applications. Mathematische Annalen 54: 125-201.

Sauer, T. 2014. Marcel Grossmann and his contribution to the general theory of relativity. In R. T. Jantzen, K. Rosquist, and R. Ruffini, eds., Proceedings of the 13th Marcel Grossmann Meeting on Recent Developments in Theoretical and Experimental General Relativity, Gravitation, and Relativistic Field Theory. Singapore: World Scientific. [arXiv:1312.4068]. 
Stachel, J. 2007. The First Two Acts. Vol. 1. Einstein's Zurich Notebook: Introduction and Source of Janssen et al. (2007a), pp. 81-112.

Veblen, O. 1927. Invariants of Quadratic Differential Forms. Cambridge University Press.

Wright, J. E. 1908. Invariants of Quadratic Differential Forms. New York: Hafner Publishing Co. 